\title{
Overconfidence in financial knowledge associated with financial risk tolerance in older adults
}

\author{
Gregory R. Samanez-Larkin \\ Duke University
}

Gary R. Mottola

Financial Industry Regulatory Authority (FINRA) Investor Education Foundation

\author{
Darby E. Heflin
}

Duke University

\author{
Lei Yu and Patricia A. Boyle \\ Rush Alzheimer's Disease Center, Rush University Medical Center
}

Author Note: Financial support for this research was provided by the FINRA Investor Education Foundation and National Institute on Aging grants R01-AG033678, R01-AG017917, and R01AG034374. Pre-registration available here: https://osf.io/2cphs and analysis scripts available here: https://osf.io/ze8gt/ Correspondence should be addressed to: Gregory Samanez-Larkin, 308 Research Drive, Box 90999, Center for Cognitive Neuroscience, Duke University, Durham, NC 27708, g.samanezlarkin@duke.edu 


\begin{abstract}
Taking excessive financial risk in older age can have harmful, far-reaching consequences as opportunities to recover lost wealth are limited. Better understanding the mechanisms of financial risk taking in older age is critically important for both identifying vulnerabilities in certain older adults and for developing interventions to empower aging investors to make wise financial choices into the most advanced ages. The goals of the present study were to identify age differences in financial literacy, confidence in financial knowledge, and risk taking and how literacy and confidence were related to financial risk taking across older adults with and without cognitive impairment (ages 58-101). Using cross-sectional data from the Rush Memory and Aging Project, analyses revealed that risk aversion was higher and self-reported willingness to take financial risks was lower at older ages. Financial literacy was similar across the sixties and seventies but lower at the oldest ages. However, confidence in financial knowledge was not associated with age when controlling for financial literacy. In exploratory analyses, a measure of overconfidence in financial knowledge was positively associated with self-reported financial risk tolerance but not a behavioral measure of risk aversion. The overconfidence effect on risk tolerance did not vary across individuals with no cognitive impairment or Mild Cognitive Impairment (MCI). Overconfidence accounted for about $6 \%$ of the variance in financial risk tolerance. The present results suggest that overconfidence may contribute to risky financial behavior. Calibration of confidence levels to actual literacy is a potential target for future interventions aimed at protecting senior investors.
\end{abstract}

Keywords: aging, financial literacy, confidence, risk taking, decision making 
Overconfidence in financial knowledge associated with financial risk tolerance in older adults

Older adults manage an increasing share of national wealth in the United States and other graying nations. Risky decisions by aging investors may have effects on financial markets in general but certainly have critical effects on the long-term health and well being of the individual decision maker. Taking excessive financial risk in older age can be devastating as opportunities to recover lost wealth are limited as an individual ages. The most extreme examples are individuals who lose a lifetime of financial savings to scams or fraud (Deevy et al., 2012; DeLiema et al., 2017; Pillemer et al., 2015; Spreng et al., 2016). Many researchers have been studying individual differences in financial decision making across adulthood to identify potential risk factors (personal or situational) or profiles of individuals who may be most vulnerable to scams and fraud (Spreng et al., 2016). Although a fraudulent investment may be one of the worst financial mistakes an older adult can make, there are many opportunities to take excessive financial risk even with legitimate investments. A better understanding of the factors that contribute to financial risk taking in older age is critically important for both identifying vulnerabilities in certain older adults and for developing interventions to empower aging investors to make wise financial choices into the most advanced ages.

Behavioral and neuroimaging research has examined adult age differences in financial risk taking in an attempt to better understand whether older adults are more or less likely to take financial risks in general (Samanez-Larkin \& Knutson, 2015). Laboratory studies using behavioral tasks that assess financial risk taking have not reliably identified adult age differences (Mata et al., 2011; Rolison et al., 2013). These tasks assess risk preferences via revealed preferences from choices among hypothetical or real financial gambles or games where an individual can earn points or money. In some studies using these tasks older adults take more risk compared to younger adults, take less risk, or don't differ from younger adults. Some have suggested that the cognitive demands (e.g., learning and memory) or reward-optimizing strategies (e.g., whether it's optimal to be risk seeking or risk averse) specific to each task account for the heterogeneity of age effects across studies (Mata et al., 2011). Nevertheless, the heterogeneity of effects suggests that a single behavioral task may not provide a reliable estimate of adult age differences in risk taking (Frey et al., 2017; Mamerow et al., 2016). Overall, these studies suggest that older adults may not be more or less likely to take risks compared to younger adults. 
In contrast to the literature using behavioral tasks, studies that use self-reported measures of risk taking and risk tolerance have consistently identified adult age differences such that older adults say they take fewer risks than younger adults (Mamerow et al., 2016). These effects have been confirmed in large cross-sectional and longitudinal samples (Josef et al., 2016). Overall, these studies suggest older adults in general report being less likely to take a financial risk in their own everyday lives. Although these effects are consistent, the size of the age effects vary such that even for the highest estimated age effects, the majority of the variance in risk taking is unaccounted for by age. That is, there are many older adults that self-report that they are highly risk tolerant even in samples where the oldest adults are not risk tolerant on average. What accounts for these individual differences within older age? Although many studies have identified individual differences in cognition, personality, financial literacy, and brain structure and function related to decision making across adulthood (Eppinger et al., 2012; Hämmerer \& Eppinger, 2012; Henninger et al., 2010; Hess et al., 2015; Kircanski et al., 2018; Löckenhoff \& Samanez-Larkin, 2020; Mather, 2006; Peters et al., 2007; Samanez-Larkin \& Knutson, 2015; Seaman et al., 2017; Shao \& Lee, 2014; Spreng et al., 2016, 2017; Strough et al., 2011), fewer studies have focused on individual differences within older age (Boyle, Yu, Buchman, et al., 2012; Boyle, Yu, Wilson, et al., 2012; Han, Boyle, Arfanakis, et al., 2016; Han, Boyle, James, et al., 2016; Han et al., 2014). There is some evidence that different cognitive mechanisms account for individual differences in financial decision making during younger and older adulthood (Agarwal et al., 2009; Li et al., 2013). Although there are a couple of exceptions, nearly all studies of young to older adulthood have been underpowered to detect individual differences within any specific age group. Given the consequences of risky decisions, it is critically important to better understand mechanisms of financial risk taking in older age.

The present study examined mechanisms of individual differences in financial decision making within older adulthood. Pre-dating nearly all of the studies of aging and risk taking were studies of cautiousness, some of which suggested that older adults were more likely to be cautious in responding on motor tasks (Calhoun \& Hutchison, 1981; Okun, 1976). Many early studies of risk aversion in older age cited this work as a potential more domain general mechanism. It was never clarified whether motor cautiousness is related to avoidance of risk. However, this earlier work on cautiousness does raise questions about the role of confidence on financial risk taking. Although confidence is regularly measured in studies of age differences in 
learning and memory, it has been mostly ignored in studies of aging and decision making. Across studies of confidence in learning, memory, and knowledge, there are not clear age differences (Dahl et al., 2009; Dodson et al., 2007, 2015; Kovalchik et al., 2005; Marquié \& Huet, 2000; Pliske \& Mutter, 1996; Serra et al., 2008). That is, it appears that confidence levels are similar across adult age groups. Many studies have documented overconfidence in knowledge in non-experts of all ages (Camerer \& Lovallo, 1999). One study of aging shows weaker effects of overconfidence in knowledge in older adults, who in general score higher on tests of general knowledge (Kovalchik et al., 2005) but other studies show no age differences (Dahl et al., 2009).

Whether confidence differs across adulthood or not, individual differences in confidence may affect decision making. Studies in younger and middle-aged adults suggest that overconfidence increases financial risk taking (Broihanne et al., 2014; Minggui et al., 2013; Nosić \& Weber, 2010). At least one study of older adults showed that although cognitive decline was associated with a decline in confidence in general and a decline in financial literacy, cognitive decline was not associated with a decline in confidence in financial knowledge (Gamble et al., 2015). Studies have not examined how both literacy and confidence in financial knowledge may be related to financial risk taking among older adults.

The goals of the present study were to identify (1) age differences in financial literacy, confidence in financial knowledge, and risk taking, (2) how both literacy and confidence were related to financial risk taking, and (3) how these individual difference effects might vary across age or clinical cognitive status (MCI, AD). In a pre-registered analysis plan, we expected age to be weakly, positively correlated with preferences for smaller, certain rewards over larger, risky rewards (i.e., risk aversion) and weakly, negatively correlated with preferences for smaller, sooner rewards over larger, later rewards (i.e., time discounting). We expected age to be weakly, negatively correlated with financial literacy with both linear and quadratic age effects such that literacy is similar across younger-old ages but lower at the oldest ages. We expected literacy but not age to be correlated with confidence in financial knowledge. We expected an interaction between literacy and confidence such that individuals with low literacy would have a stronger effect of confidence on risk aversion. We did not expect the same interaction between literacy and confidence on time discounting. We expected a three-way interaction such that the interaction between confidence and literacy would be strongest at the oldest ages. Finally, we 
expected the literacy by confidence interactions on risk aversion to be strongest in the MCI and AD groups.

\section{Method}

Data analyzed came from the Rush Memory and Aging Project (MAP). The MAP is an epidemiologic cohort study of common chronic conditions of aging. Older adults are recruited from retirement communities and subsidized senior housing facilities throughout Chicagoland and northeastern Illinois without known dementia at enrollment. All participants agree to annual clinical evaluation, cognitive testing. The MAP began in 1997 and a substudy on decision making started in 2010. The present data were from the first complete decision making assessment for each MAP participant. The present data were from the first complete decisionmaking assessment (Boyle et al., 2011) for each MAP participant. A script that extracts these cross-sectional data from the complete longitudinal dataset for each participant as of October 2019 is available at: https://osf.io/ze8gt/

A data request was submitted through the Rush Alzheimer's Disease Center Research Resource Sharing Hub (https://www.radc.rush.edu/). The analysis plan and hypotheses were preregistered on September 5, 2019 (https://osf.io/2cphs) and the data were received on October 1, 2019. Demographic data, global cognitive function, clinical cognitive diagnosis, and all variables from the decision making battery were requested. Additional affect and personality variables were included in the initial request but never analyzed. Detailed documentation for all variables are provided here: https://www.radc.rush.edu/docs/var/variables.htm

Demographics. Demographic data include sex, years of education, income, race (White, Black or African American, American Indian or Alaska Native, Native Hawaiian or Other Pacific Islander, Asian, Other, or Unknown), Spanish/Hispanic/Latino origin, marital status, and age. The sample included for the present analyses was $93.3 \%$ White, 5.3\% Black, 1.4\% American Indian/Native, Asian, Other, or Unknown. Three percent were of Spanish/Hispanic/Latinx ethnicity. The average annual income of participants included in these analyses was around $\$ 35,000$ (nearly all participants are retired) and average education was nearly 16 years, so it was a mostly college-educated sample. See Table 1 for descriptive statistics. 
Table 1. Sample characteristics. Means (and standard deviations) for demographic and decision making-related variables for all participants and for subgroups by clinical cognitive diagnosis. Counts for sex. Medians (and inter-quartile ranges) for education and income.

\begin{tabular}{lcccc} 
& All & NCI & MCI & AD \\
& $(\mathrm{N}=1242)$ & $(\mathrm{N}=911)$ & $(\mathrm{N}=262)$ & $(\mathrm{N}=53)$ \\
\hline Age & $81.58(7.65)$ & $80.46(7.71)$ & $84.44(6.37)$ & $86.63(6.96)$ \\
Sex & $938 \mathrm{~F} / 304 \mathrm{M}$ & $706 \mathrm{~F} / 205 \mathrm{M}$ & $186 \mathrm{~F} / 76 \mathrm{M}$ & $36 \mathrm{~F} / 17 \mathrm{M}$ \\
Education & $16(5)$ & $16(5)$ & $16(5)$ & $16(3)$ \\
Income & $8(3)$ & $8(4)$ & $8(4)$ & $8(4)$ \\
Global cognition & $0.13(0.62)$ & $0.35(0.44)$ & $-0.36(0.46)$ & $-1.31(0.62)$ \\
Risk aversion & $0.31(0.31)$ & $0.30(0.30)$ & $0.36(0.32)$ & $0.39(0.35)$ \\
Financial risk & $4.98(2.14)$ & $5.05(2.06)$ & $4.70(2.30)$ & $5.10(2.61)$ \\
tolerance & $0.34(0.41)$ & $0.32(0.40)$ & $0.37(0.43)$ & $0.48(0.45)$ \\
Time discounting & $74 \%(16 \%)$ & $76 \%(15 \%)$ & $69 \%(17 \%)$ & $57 \%(15 \%)$ \\
Financial literacy & & & & \\
Confidence in & $3.14(0.56)$ & $3.17(0.55)$ & $3.06(0.59)$ & $2.86(0.55)$ \\
financial & & & & \\
knowledge & $-0.009(.99)$ & $-0.09(.94)$ & $0.14(1.03)$ & $0.55(1.13)$ \\
Overconfidence & & & & \\
\hline & & & &
\end{tabular}

$\mathrm{NCI}=$ no cognitive impairment, $\mathrm{MCI}=$ Mild Cognitive Impairment, AD = Alzheimer's Disease . $\mathrm{N}$ is maximum observations; some variables are missing data as indicated in the reporting of results. Education is number of years completed. Income is a 10-category ordinal variable with 8 corresponding to an annual income of $\$ 35,000-\$ 49,999$. Global cognition is an average of $19 \mathrm{z}$ scored cognitive tests. Risk aversion is the gamma coefficient from mixed gamble choices. Financial risk tolerance is a self-report on a 10-point scale with 10 being complete willingness to take financial risk. Time discounting is the hyperbolic discount rate estimated from choices between smaller, sooner rewards and larger, later rewards. Financial literacy is a percentage accuracy score. Confidence is on a 1-4 scale with 3 being fairly confident and 4 being extremely confident. Overconfidence is z-scored confidence minus z-scored literacy based on whole sample means and standard deviations. 
Cognitive diagnosis. Clinical diagnosis of cognitive status is determined at each assessment based on a three-stage process including computer scoring of cognitive tests, clinical judgment by a neuropsychologist, and diagnostic classification by a clinician. Unless otherwise specified, analyses of the full sample included all individuals (no cognitive impairment, MCI, $\mathrm{AD}$, and other dementia). Specific analyses of subgroups in the present study examined effects within individuals with NCI, MCI and AD but not individuals with other dementia. The AD group was excluded from analyses of self-reported financial risk tolerance due to the unreliability of self-reports in AD.

Cognition. A measure of global cognitive function is computed from an average of $\mathrm{z}$ standardized scores from a battery of 19 cognitive tests that include measures of episodic memory, semantic memory, working memory, perceptual orientation, and perceptual speed.

Decision making. All variables from the decision making battery were requested, but the only variables used for analyses were risk tolerance, risk aversion, temporal discounting, financial literacy, confidence in financial knowledge, scam susceptibility, and fraud victimization.

Financial risk tolerance is a self-report measure of willingness to take risks. Participants are asked to rate the statement, "What is or has been over your lifetime your willingness to take risks in financial matters?" on a scale from 1 to 10 with 1 indicating to a complete unwillingness to take risks and 10 indicating a complete willingness to take risks. A higher score relates to greater willingness to take risks.

Risk aversion is a measure of the tendency to prefer a certain but smaller monetary reward over a risky but possibly larger one. Risk aversion is assessed with 10 questions that ask participants if they would hypothetically prefer $\$ 15$ for sure OR a coin toss in which they could get $\$[$ an amount greater than $\$ 15]$ if they flip heads or nothing if they flipped tails. Possible gains range from $\$ 20$ to $\$ 300$ and gain amounts vary across questions. The risk aversion coefficient, gamma, is estimated based on the participant's odds of choosing the gamble over the safe option and the expected utility of the gamble for each question (Boyle et al., 2011). Higher scores correspond to more risk aversion (i.e., less likely to choose a risky reward).

Temporal discounting is assessed with 10 questions that ask participants if they would prefer a smaller monetary reward now or a larger reward later. Five questions ask about \$10 now compared to $\$ 14, \$ 20, \$ 15, \$ 11, \$ 18, \$ 13$, or $\$ 30$ in a month and five questions ask about $\$ 1000$ 
now compared to $\$ 1200, \$ 1100, \$ 2200, \$ 3000$, or $\$ 1500$ in a year. The hyperbolic time discount rate, alpha, is estimated separately for the $\$ 10$ now questions and the $\$ 1000$ now questions. Higher scores mean a participant is less likely to wait for a larger, later reward. As specified in the pre-registration, we created an average measure of time discounting by averaging the two discount rates.

Financial literacy is assessed with 23 questions designed to measure knowledge of financial information and concepts and numeracy. Questions assess knowledge of financial terms, institutions, and stocks and bonds (e.g., "A mutual fund is an investment that holds what?: Only stocks; Only bonds; Stocks AND bonds." and "When interest rates go up, what do bond prices do?: Go down; Go up; Stay the same”). Questions dealing with numeracy assess the ability to perform simple monetary calculations such as sales (e.g., "A store is offering $15 \%$ off a television that is normally priced at $\$ 1000$. How much money would you save on the TV during this sale?: \$15; \$150; \$1500”) and interest rates (e.g., "Suppose you had \$100 in a savings account and the interest rate was $2 \%$ per year. After 5 years, how much do you think you would have in the account if you left the money to grow?: More than \$102; Exactly \$102; Less than \$102”). All answer choices are multiple choice or true/false with only one correct answer. The score is the total percentage correct.

Confidence in financial knowledge is based on the average of 9 questions. Specifically, after answering a financial knowledge question, participants are asked, "How confident are you that you answered that question correctly?" on a 4-point rating scale $(1=$ Not at all confident, $2=$ A little confident, 3 = Fairly confident, $4=$ Extremely confident) with a higher rating indicating greater confidence. Hereafter this variable it referred to as confidence in financial knowledge.

Scam susceptibility is measured using a self-report scale based on content from AARP and questions from the Financial Industry Regulatory Authority's Risk Meter. Participants are asked to rate their agreement with 5 statements (e.g., "I feel I have to answer the phone whenever it rings, even if I do not know who is calling." and "If something sounds too good to be true, it usually is.”) using 7-point Likert scales (from $1=$ Strongly agree to $7=$ Strongly disagree). Some items are reverse coded and the overall scam susceptibility measure is the average rating of individual items so that higher scores indicate greater susceptibility to scams. 
Fraud victimhood is a dichotomous variable for which participants respond to the question, "In the past year, were you a victim of financial fraud or have you been told you were a victim of financial fraud?" with Yes or No.

Descriptive statistics for all decision making variables used for analyses in the present study are presented in Table 1. All regression analyses reported used z-standardized versions of the independent and dependent variables (with the exception of the dichotomous fraud dependent variable) to obtain standardized regression coefficients $\beta$ and corresponding $95 \%$ confidence intervals (CI). Significant bivariate associations were followed with analyses that included covariates to assess the robustness of effects. If the bivariate associations were non-significant, no additional analyses were performed. It should be noted that with a sample size over 1000, many very small effects are statistically significant at $\mathrm{p}<.05$. Thus, the reporting of results and discussion are focused primarily on effect sizes. Commented analysis scripts and markdown files are publicly available at: https://osf.io/ze8gt/

\section{Results}

Preregistered analyses. Consistent with our hypotheses, there was a weak, positive linear association between age and risk aversion, $\beta=.13,95 \%$ CI $[.07, .19], p<.001$ (Figure 1), and a non-significant quadratic association, $\beta=.02,95 \% \mathrm{CI}[-.02, .06], p=.355$. Older individuals were more likely to choose safe options and avoid a risky gamble. Inconsistent with our hypotheses, there was a weak, positive linear association between age and time discounting, $\beta=.10,95 \% \mathrm{CI}[.04, .15], p=.001$, and a non-significant quadratic association, $\beta=.001,95 \%$ CI $[-.04, .04], p=.973$. Older individuals were more likely to choose smaller, sooner rewards than larger, later rewards.
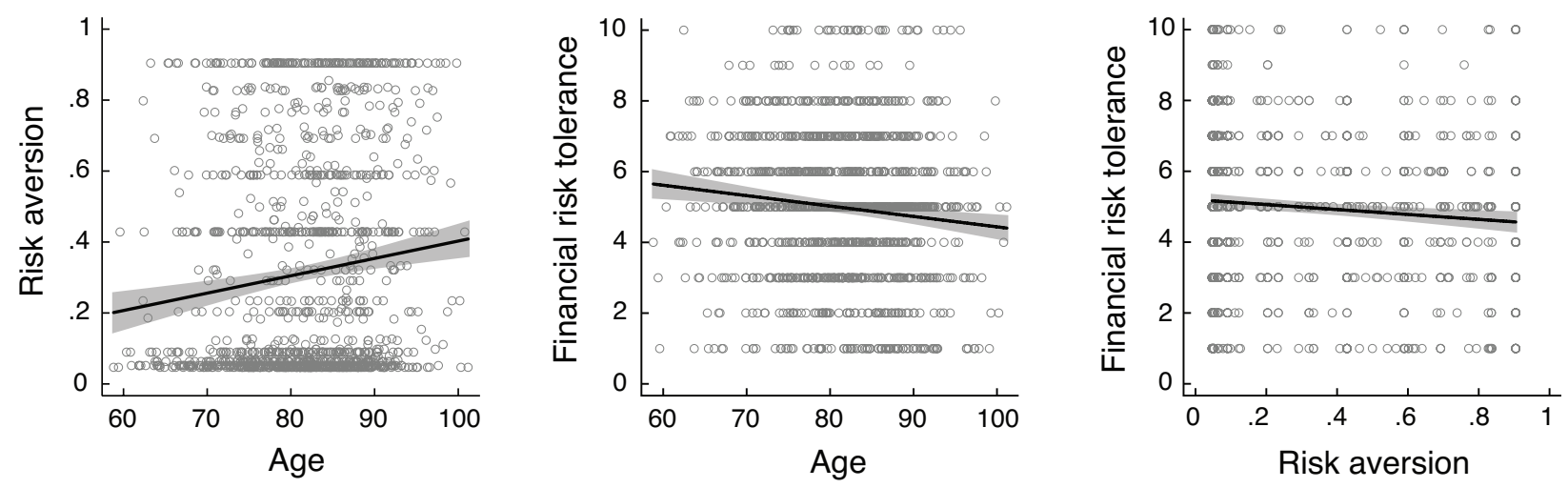
Figure 1. Associations between age and risk aversion and financial risk tolerance. Lines with shaded error are best fit (linear) regression lines from bivariate associations without covariates. Shaded regions indicate $95 \%$ confidence intervals around best fit line. Results reported in text use z-standardized variables but unstandardized variables are displayed here to show original units.

Consistent with our hypotheses, there was a negative linear association between age and financial literacy, $\beta=-.27,95 \% \mathrm{CI}[-.33,-.21], p<.001$, and a significant quadratic association between age and financial literacy, $\beta=-.08,95 \% \mathrm{CI}[-.12,-.04], p<.001$ (Figure 2). with the age association weakest in the sixties and early seventies and strongest from the late seventies until age 101. There was a weak, negative linear association between age and confidence in financial knowledge, $\beta=-.16,95 \% \mathrm{CI}[-.22,-.11], p<.001$ (Figure 2), and a non-significant quadratic association, $\beta=-.03,95 \% \mathrm{CI}[-.07, .01], p=.150$. The oldest individuals were the least confident in their financial knowledge. Consistent with our hypotheses, after controlling for financial literacy, the effect of age on confidence was no longer significant, $\beta=-.04,95 \% \mathrm{CI}[-$ $.09, .01], p=.163$, and the effect of literacy on confidence was strong and positive, $\beta=.51,95 \%$ CI $[.45, .56], p<.001$. Individuals with higher financial literacy had higher levels of confidence in their knowledge.
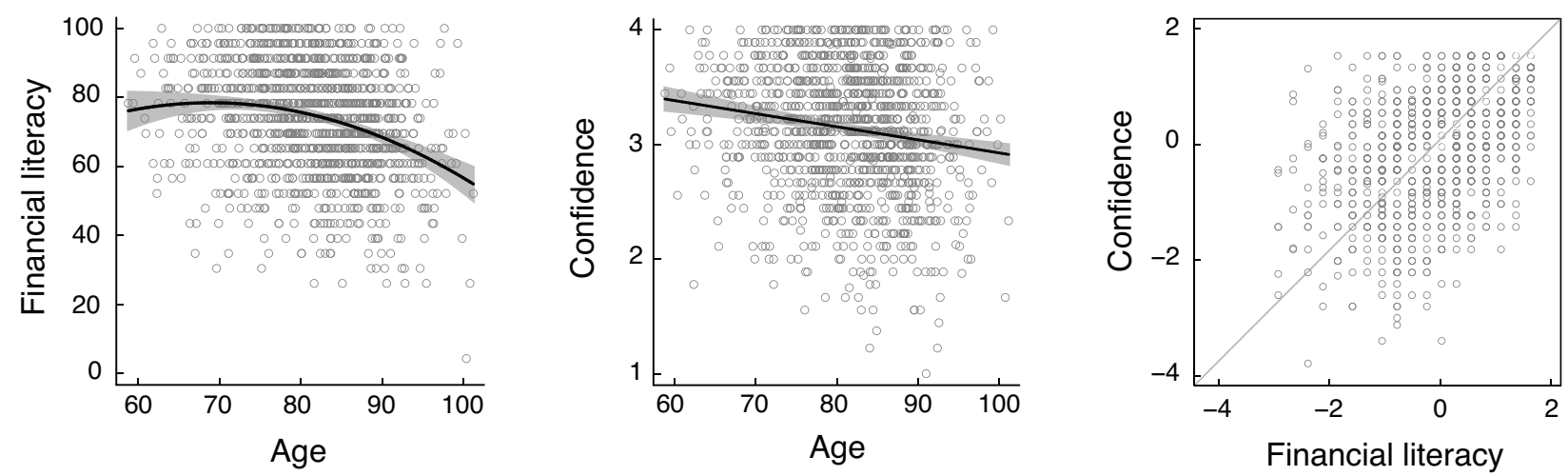

Figure 2. Associations between age and financial literacy and confidence in financial knowledge. Lines with shaded error are best fit (linear or quadratic) regression lines from bivariate associations without covariates. Shaded regions indicate $95 \%$ confidence intervals around best fit line. Gray diagonal in the scatterplot of confidence and literacy is a unity line depicting the relative distribution of individuals with confidence well calibrated to literacy (close to line), overconfident relative to actual literacy (above unity line), and underconfident relative to actual literacy (below unity line). Results reported in text use z-standardized variables but 
unstandardized variables are displayed in the plot on the left and middle to show original units. The plot of the right uses z-standardized variables.

Inconsistent with our hypotheses, there were no significant interactions between literacy and confidence on risk aversion and the effects did not vary substantially across diagnostic groups (Table 2). Although not significant, there was a stronger effect of confidence and weaker effect of literacy on risk aversion in the AD group compared to the no cognitive impairment and MCI groups. There were no interactions between literacy and confidence on time discounting as predicted.

Table 2. Main and interaction effects of confidence, literacy, and age on risk aversion and time discounting.

RISK AVERSION

$\begin{array}{llllll}\text { All } & \mathrm{NCI} & \mathrm{MCI} & \mathrm{AD} & \mathrm{All} & \mathrm{NCI}\end{array}$

\begin{tabular}{|c|c|c|c|c|c|c|c|c|}
\hline & All & NCI & MCI & $\mathrm{AD}$ & All & $\mathrm{NCI}$ & MCI & $\mathrm{AD}$ \\
\hline Confidence & -.04 & -.04 & -.08 & .33 & -.05 & $-.10 *$ & -.01 & -.23 \\
\hline Literacy & $-.19 * *$ & $-.20 * *$ & $-.16^{*}$ & -.03 & $-.22 * *$ & $-.19 * *$ & $-.19 *$ & -.17 \\
\hline $\begin{array}{l}\text { Confidence } \\
\text { X literacy }\end{array}$ & -.03 & -.03 & -.07 & .09 & -.01 & .04 & -.04 & -.37 \\
\hline Age & $.09 *$ & .07 & .14 & .20 & .03 & .02 & .06 & -.38 \\
\hline $\begin{array}{l}\text { Confidence } \\
\mathrm{X} \text { age }\end{array}$ & $.07 *$ & $.08 *$ & .05 & .35 & .001 & -.02 & .07 & .04 \\
\hline $\begin{array}{l}\text { Literacy X } \\
\text { age }\end{array}$ & $-.10 *$ & $-.09 *$ & -.13 & .05 & .06 & .07 & -.04 & -.06 \\
\hline Confidence & & & & & & & & \\
\hline $\begin{array}{l}\text { X literacy X } \\
\text { age }\end{array}$ & -.04 & -.04 & -.03 & -.02 & .02 & .04 & .05 & .08 \\
\hline
\end{tabular}

*Significant at $p<.05$. **Significant at $p<.001$. All $(\mathrm{N}=1,221), \mathrm{NCI}=$ no diagnosis of cognitive impairment $(\mathrm{N}=903), \mathrm{MCI}=$ Mild Cognitive Impairment $(\mathrm{N}=258), \mathrm{AD}=$ Alzheimer's dementia $(\mathrm{N}=47)$ 
Exploratory analyses. Given the lack of interaction between literacy and confidence, more exploratory (non-pre-registered) analyses examined effects of overconfidence relative to actual literacy. A scatterplot of literacy and confidence revealed that although many individuals had confidence levels relatively well calibrated to their literacy levels, there was variation in over- and under-confidence relative to actual literacy (Figure 2). A measure of overconfidence was computed by subtracting z-standardized literacy scores from z-standardized confidence scores. On average across the sample, individuals were not over- or under-confident relative to their literacy levels as indicated by a non-significant difference between overconfidence scores and zero, $t_{1222}=-0.328, \mathrm{p}=.743$.

There was a weak, positive linear association between age and overconfidence, $\beta=.10$, $95 \%$ CI $[.04, .15], p=.001$, but a non-significant quadratic association, $\beta=.04,95 \%$ CI $[-.002$, $.08], p=.060$. However, the age association with overconfidence was non-significant, $\beta=-.04$, $95 \%$ CI $[-.09, .01], p=.163$, after controlling for literacy as in the analyses of confidence reported above. There was a weak, positive association between overconfidence and risk aversion, $\beta=.08,95 \% \mathrm{CI}[.02, .13], p=.008$, controlling for age, but the effect was nonsignificant, $\beta=-.03,95 \% \mathrm{CI}[-.09, .03], p=.351$, after also controlling for literacy and global cognition. Similarly, there was a weak, positive association between overconfidence and time discounting, $\beta=.08,95 \% \mathrm{CI}[.02, .13], p=.007$, controlling for age, but the association was non-significant, $\beta=-.06,95 \%$ CI $[-.12, .01], p=.073$, after also controlling for literacy and global cognition.

Additional exploratory analyses examined the potential effects of overconfidence on other risky financial behaviors such as self-reported financial risk tolerance, scores on a selfreport measure of scam susceptibility, and history of fraud victimization. As self-reported financial risk tolerance was not a variable included in the pre-registered analyses, we first examined effects of age. Conceptually consistent with our pre-registered hypotheses about aging and risk aversion, there was a weak, negative association between age and self-reported financial risk tolerance, $\beta=-.12,95 \% \mathrm{CI}[-.17,-.06], p<.001$ (Figure 1), and a non-significant quadratic association, $\beta=-.03,95 \% \mathrm{CI}[-.08, .01], p=.111$. The oldest adults reported a lower willingness to take financial risk. Self-reported financial risk tolerance was only weakly associated with risk aversion, $\beta=-.09,95 \% \mathrm{CI}[-.15,-.04], p=.001$, controlling for age. The 
effect size was the same when not controlling for age, $\beta=-.10,95 \% \mathrm{CI}[-.16,-.05], p<.001$ (Figure 1).

There was a weak, positive association between overconfidence and self-reported financial risk tolerance in everyday life, $\beta=.15,95 \% \mathrm{CI}[.09, .20], p<.001$, controlling for age, that remained significant and was stronger, $\beta=.24,95 \% \mathrm{CI}[.18, .30], p<.001$, after also controlling for financial literacy, risk aversion, and global cognition. The association of overconfidence and financial risk tolerance did not differ across individuals without a cognitive diagnosis and individuals with MCI (see Table 3 and Figure 3). Individuals with AD were not included in these analyses given the lack of reliability of their self-reports.
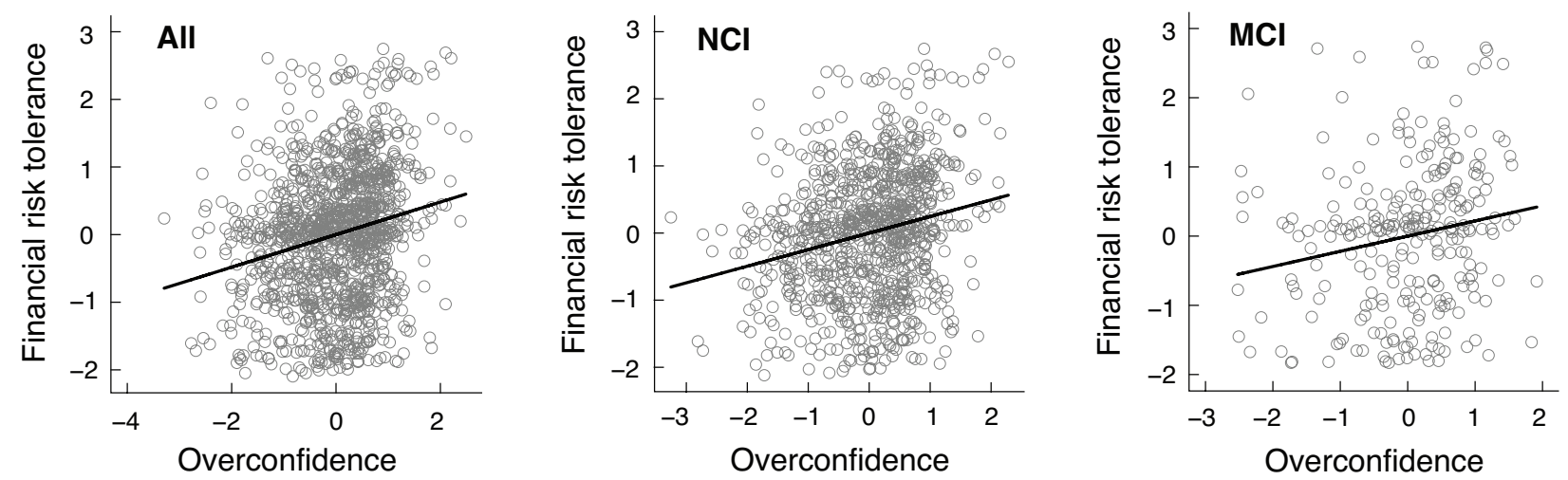

Figure 3. Associations between overconfidence and financial risk taking (partial regression plots controlling for co-variates) displayed for all participants (ALL) and for the subset of individuals with no diagnosis of cognitive impairment (NCI) or with Mild Cognitive Impairment (MCI).

Table 3. Effects of overconfidence on self-reported financial risk taking in everyday life controlling for literacy, risk aversion, age, and global cognition.

FINANCIAL RISK TOLERANCE

All NCI MCI

\begin{tabular}{lccc}
\hline Overconfidence & $.24 *[.18, .30]$ & $.25^{*}[.18, .32]$ & $.22 *[.08, .36]$ \\
Financial literacy & $.19 *[.12, .27]$ & $.19^{*}[.11, .27]$ & $.23 *[.07, .39]$ \\
Risk aversion & $-.08^{*}[-.13,-.02]$ & $-.07 *[-.13,-.01]$ & $-.03[-.16, .10]$ \\
Age & $-.08^{*}[-.14,-.02]$ & $-.10^{*}[-.17,-.04]$ & $.02[-.14, .18]$ \\
Global cognition & $-.03[-.10, .04]$ & $-.05[-.15, .05]$ & $-.03[-.23, .18]$ \\
\hline
\end{tabular}


*Significant at $p<.05$. All $(\mathrm{N}=1,215), \mathrm{NCI}=$ no diagnosis of cognitive impairment $(\mathrm{N}=901)$, $\mathrm{MCI}=$ Mild Cognitive Impairment $(\mathrm{N}=258)$

Overconfidence was not related to fraud victimization, $\beta=.20,95 \% \mathrm{CI}[-.02, .43], p=$ .067 , controlling for age $(\mathrm{N}=1219)$. Being one standard deviation higher on overconfidence was associated with only about a $1 \%$ increase in fraud victimization. Overconfidence was very weakly, positively related to scam susceptibility, $\beta=.06,95 \%$ CI $[.01, .11], p=.026$, controlling for age $(\mathrm{N}=1219)$, but the sign of that effect reversed, $\beta=-.07,95 \%$ CI $[-.13,-.01], p=.014$, after also controlling for financial literacy and global cognition $(\mathrm{N}=1215)$.

\section{Discussion}

The present study investigated age differences in financial literacy, confidence in financial knowledge, and risk taking among older adults with and without cognitive impairment and the potential contributions of individual differences in literacy and confidence on financial risk taking. Across groups, pre-registered and exploratory analyses identified relatively small adult age differences in risk aversion, financial risk tolerance, and financial literacy. There were substantial individual differences in risk taking that were independent of age. The most novel results were based on exploratory analyses and revealed that individuals who had high confidence in their financial knowledge relative to their actual financial literacy (i.e., overconfidence) reported taking more financial risks in everyday life. The effect of overconfidence on financial risk taking did not vary between healthy adults and the MCI or AD subgroups and was independent of age and global cognition.

Effects of age on financial literacy revealed that literacy (a measure of both financial knowledge and numeracy) was similar among sixty- and seventy-year-olds but was significantly lower in eighty-year-olds and even lower in ninety-year-olds. To some, this effect may seem surprising given widespread evidence that general knowledge (not specific to financial knowledge) increases and does not seem to decline over most of adulthood. Other studies with large samples that span young to older adulthood have documented that financial knowledge is lowest in young adulthood and higher in middle and older age (Li et al., 2013,2015) with potential non-linear effects such that knowledge plateaus in midlife (Agarwal et al., 2009). 
Importantly, the age range of this sample was 58 to 101 . The present sample provides updated cross-sectional evidence that at the oldest stages of adulthood, financial literacy may decline. Earlier analyses of a subset of these data that did not include the MCI or AD groups revealed a similar pattern and additional longitudinal analyses revealed that declines in cognition, particularly episodic memory and executive function, preceded declines in financial literacy but not word knowledge (Boyle et al., 2013). Other studies using independent data sets have also documented declines in financial literacy in older age (Finke et al., 2016). It is likely that differences between studies are due to differences in the segments of adulthood measured, the approach (comparisons of groups of individuals at different ages vs. longitudinal studies) and the particular assessments of financial literacy. Performance on some measures of financial literacy, especially those used here with numeracy assessments, have higher cognitive demands than others (Li et al., 2013, 2015).

Despite age differences in financial literacy, there were not consistent age differences in confidence in financial knowledge after controlling for actual financial literacy. This is consistent with the literature on aging and confidence in learning, memory, and knowledge which does not show consistent adult age differences (Dahl et al., 2009; Dodson et al., 2007, 2015; Marquié \& Huet, 2000; Pliske \& Mutter, 1996; Serra et al., 2008). On average, individuals' confidence levels were well calibrated to their actual levels of literacy. However, despite financial literacy and confidence being strongly correlated, there were many individuals with high confidence relative to their literacy (overconfident) and low confidence relative to their literacy (underconfident). In exploratory analyses, a measure of overconfidence in financial knowledge was positively correlated with self-reported financial risk tolerance but not other traditional decision-making measures of risk aversion or time discounting (which may in part index impulsivity) after controlling for actual literacy. The association between overconfidence and risk tolerance is conceptually consistent with several prior studies of overconfident financial traders, business managers, and corporate executives taking greater risks (Broihanne et al., 2014; Minggui et al., 2013; Niu, 2010; Nosić \& Weber, 2010; Odean, 1998).

Overconfidence was more consistently related to the self-report measure of financial risk tolerance than the more behavioral measure of risk aversion. In fact, the behavioral and selfreport measures were very weakly correlated. This is consistent with many prior studies which have demonstrated weak correlations between self-report and behavioral measures in general 
(Dang et al., 2020), between self-report and behavioral measures of risk (Frey et al., 2017), and differential associations between risk measures and age (Mamerow et al., 2016; Mata et al., 2011; Rolison et al., 2013). Specifically, associations between age and more behavioral measures of risk aversion (like the one used here based on a series of choices between mixed gambles) have been highly inconsistent across the literature with studies showing age-related increases in risk aversion, decreases, or no age differences (Mata et al., 2011; Mather, 2006; Rolison et al., 2013; Samanez-Larkin \& Knutson, 2015; Shao \& Lee, 2014). In contrast, self-report measures of risk taking show consistent negative associations with age in cross-sectional and longitudinal studies (Josef et al., 2016). That is, while older adults do not always reveal risk averse preferences in their choices on a laboratory task, when asked about their preferences for risk they reliably state that they are less willing to take risks than younger adults in general and than they themselves took as younger adults. In the present study, the measure of overconfidence was more strongly and robustly correlated with the measure of financial risk taking that shows the most reliable age effects in the literature.

It is important to note that the overconfidence effect on financial risk tolerance was independent of age. The finding is somewhat inconsistent with prior research documenting an age-related increase in overconfidence accounting for some older individuals having riskier retirement portfolios (Pak \& Chatterjee, 2016). In the present study, overconfidence did not explain age differences in financial risk tolerance; the small effect of age on financial risk tolerance was only slightly weakened by controlling for overconfidence. Importantly, the inconsistency between the present study and past research was in the finding of age differences in overconfidence. However, both studies are consistent in documenting individual difference associations between overconfidence and financial risk taking in older adulthood. In the present study, across four decades of older adulthood, the most overconfident individuals reported being more willing to take financial risks. Age explained only about $1 \%$ of the variance in financial risk tolerance whereas overconfidence explained about $6 \%$. This is not a large effect; the vast majority of individual differences in risk tolerance were unexplained.

The overconfidence effect on financial risk tolerance was also independent of cognitive status. The effect sizes were almost identical and the confidence intervals around these effects were almost completely overlapping in individuals with no cognitive impairment and individuals with MCI. The association between overconfidence and risk tolerance did not seem to depend on 
group differences in overconfidence, risk tolerance, or cognitive status. Across the sample, the most overconfident individuals were more likely to say they were willing to take financial risks. In the current cross-sectional analyses there were no cognitive diagnostic group differences in overconfidence after controlling for actual literacy.

Related recent work with some of the same data used here reveals associations between a different measure of overconfidence and cognitive diagnosis. In longitudinal analyses, a decrease in confidence relative to literacy was associated with a higher likelihood of developing MCI and AD (Yu et al., 2020). This effect may seem in the opposite direction of the present results, however the results are not necessarily incompatible. It is possible that individuals who start to feel more uncertain about their financial knowledge or knowledge or abilities in general may be in the early stages of some form of cognitive impairment. However, the present resulst suggest that at any stage of cognitive capacity relative overconfidence may contribute to financial risk taking.

Additional exploratory analyses examined potential effects of overconfidence on scam susceptibility and fraud victimization but the weak bivariate associations were not robust to controlling for actual literacy levels or global cognition. The results suggest that subjective perceptions of confidence in financial knowledge may not be important predictors or correlates of falling victim to scams or fraud.

The current study was a secondary data analysis project for which hypotheses and analyses were pre-registered before receiving the data from Rush Alzheimer's Disease Center. Some of the pre-registered hypotheses were confirmed but the most interesting effects with literacy and confidence were based on exploratory analyses. The initial hypotheses related to the interaction between literacy and confidence were based on the assumption that confidence would have a stronger effect on the more behavioral, revealed preference measure of risk aversion when literacy was low, but the results did not reveal any interactions. The difference score used in exploratory analyses examined overconfidence effects across all literacy levels. There was no association with the risk aversion measure, but there was an association with self-reported willingness to take financial risks. The associations between overconfidence and financial risk tolerance were weak to moderate. Although the effects are conceptually related to the initial preregistered hypotheses, it should be acknowledged that they resulted from more exploratory analysis inspired by the lack of an interaction and visualizing all of the off-diagonal data in the 
scatterplot of literacy and confidence. Although the results are consistent with overconfidence effects previously reported in studies of younger adults, the effects should be replicated in an independent sample.

The present results highlight the importance of examining perceptions of confidence in financial knowledge in addition to actual financial literacy. Research on the links between financial literacy and real-world financial behaviors is mixed. One meta-analysis found that financial literacy explains a little variance in financial behaviors (Fernandes et al., 2014). Another meta-analysis found the opposite - that financial literacy is a significant predictor of financial behaviors (Kaiser \& Menkhoff, 2017). Regardless, with few exceptions, studies have mostly ignored confidence in knowledge and willingness to take financial risk (Ko \& Rapkin, 2018). Future literacy interventions may consider at least measuring confidence or changes in confidence after an intervention. Of course, confidence alone should not be a target of an intervention. Critically, the present results suggest that overconfidence could contribute to excessive financial risk taking. Confidence levels that are well calibrated to actual knowledge (Asaad, 2015) may be a more appropriate target for future interventions aimed at protecting senior investors. 


\section{References}

Agarwal, S., Driscoll, J. C., Gabaix, X., \& Laibson, D. I. (2009). The Age of Reason: Financial Decisions over the Life-Cycle with Implications for Regulation. Brookings Papers on Economic Activity, 40(2), 51-117.

Asaad, C. T. (2015). Financial literacy and financial behavior: Assessing knowledge and confidence. Financial Services Review, 24, 101-117.

Boyle, P. A., Yu, L., Buchman, A. S., \& Bennett, D. A. (2012). Risk Aversion is Associated with Decision Making among Community-Based Older Persons. Frontiers in Psychology, 3, 205. https://doi.org/10.3389/fpsyg.2012.00205

Boyle, P. A., Yu, L., Buchman, A. S., Laibson, D. I., \& Bennett, D. A. (2011). Cognitive function is associated with risk aversion in community-based older persons. $B M C$ Geriatrics, 11, 53. https://doi.org/10.1186/1471-2318-11-53

Boyle, P. A., Yu, L., Wilson, R. S., Gamble, K., Buchman, A. S., \& Bennett, D. A. (2012). Poor decision making is a consequence of cognitive decline among older persons without Alzheimer\&apos;s disease or mild cognitive impairment. PloS One, 7(8), e43647. https://doi.org/10.1371/journal.pone.0043647

Boyle, P. A., Yu, L., Wilson, R. S., Segawa, E., Buchman, A. S., \& Bennett, D. A. (2013). Cognitive decline impairs financial and health literacy among community-based older persons without dementia. Psychology and Aging, 28(3), 614-624. https://doi.org/10.1037/a0033103

Broihanne, M. H., Merli, M., \& Roger, P. (2014). Overconfidence, risk perception and the risktaking behavior of finance professionals. Finance Research Letters, 11(2), 64-73. https://doi.org/10.1016/j.frl.2013.11.002

Calhoun, R. E., \& Hutchison, S. L. (1981). Decision-making in old age: Cautiousness and rigidity. International Journal of Aging and Human Development, 13(2), 89-98.

Camerer, C., \& Lovallo, D. (1999). Overconfidence and Excess Entry: An Experimental Approach. The American Economic Review, 89(1), 306-318. JSTOR.

Dahl, M., Allwood, C. M., \& Hagberg, B. (2009). The realism in older people's confidence judgments of answers to general knowledge questions. Psychology and Aging, 24(1), 234-238. https://doi.org/10.1037/a0014048 
Dang, J., King, K. M., \& Inzlicht, M. (2020). Why Are Self-Report and Behavioral Measures Weakly Correlated? Trends in Cognitive Sciences, 24(4), 267-269. https://doi.org/10.1016/j.tics.2020.01.007

Deevy, M., Lucich, S., \& Beals, M. (2012). Scams, schemes, and swindles. Financial Fraud Research Center. http://longevity.stanford.edu/wp-content/uploads/2017/01/ScamsSchemes-Swindles-FINAL-On-Website.pdf

DeLiema, M., Mottola, G. R., \& Deevy, M. (2017). Findings from a Pilot Study to Measure Financial Fraud in the United States. Stanford Center on Longevity. https://doi.org/10.2139/ssrn.2914560

Dodson, C. S., Bawa, S., \& Krueger, L. E. (2007). Aging, metamemory, and high-confidence errors: A misrecollection account. Psychology and Aging, 22(1), 122-133. https://doi.org/10.1037/0882-7974.22.1.122

Dodson, C. S., Powers, E., \& Lytell, M. (2015). Aging, confidence, and misinformation: Recalling information with the cognitive interview. Psychology and Aging, 30(1), 46-61. https://doi.org/10.1037/a0038492

Eppinger, B., Nystrom, L. E., \& Cohen, J. D. (2012). Reduced Sensitivity to Immediate Reward during Decision-Making in Older than Younger Adults. PloS One, 7(5), e36953. https://doi.org/10.1371/journal.pone.0036953.g005

Fernandes, D., Lynch, J. G., \& Netemeyer, R. G. (2014). Financial Literacy, Financial Education, and Downstream Financial Behaviors. Management Science, 60(8), 18611883. https://doi.org/10.1287/mnsc.2013.1849

Finke, M. S., Howe, J. S., \& Huston, S. J. (2016). Old Age and the Decline in Financial Literacy. Management Science, 63(1), 213-230. https://doi.org/10.1287/mnsc.2015.2293

Frey, R., Pedroni, A., Mata, R., Rieskamp, J., \& Hertwig, R. (2017). Risk preference shares the psychometric structure of major psychological traits. Science Advances, 3(10), e1701381. https://doi.org/10.1126/sciadv.1701381

Gamble, K., Boyle, P., Yu, L., \& Bennett, D. (2015). Aging and Financial Decision Making. Management Science, 61(11), 2603-2610. https://doi.org/10.1287/mnsc.2014.2010

Hämmerer, D., \& Eppinger, B. (2012). Dopaminergic and prefrontal contributions to rewardbased learning and outcome monitoring during child development and aging. Developmental Psychology, 48(3), 862-874. https://doi.org/10.1037/a0027342 
Han, S. D., Boyle, P. A., Arfanakis, K., Fleischman, D., Yu, L., James, B. D., \& Bennett, D. A. (2016). Financial literacy is associated with white matter integrity in old age. NeuroImage, 130, 223-229. https://doi.org/10.1016/j.neuroimage.2016.02.030

Han, S. D., Boyle, P. A., James, B. D., Yu, L., Barnes, L. L., \& Bennett, D. A. (2016). Discrepancies between cognition and decision making in older adults. Aging Clinical and Experimental Research, 28(1), 99-108. https://doi.org/10.1007/s40520-015-0375-7

Han, S. D., Boyle, P. A., Yu, L., Fleischman, D. A., Arfanakis, K., Leurgans, S., \& Bennett, D. A. (2014). Financial literacy is associated with medial brain region functional connectivity in old age. Archives of Gerontology and Geriatrics, 59(2), 429-438. https://doi.org/10.1016/j.archger.2014.05.001

Henninger, D. E., Madden, D. J., \& Huettel, S. A. (2010). Processing speed and memory mediate age-related differences in decision making. Psychology and Aging, 25(2), 262-270. https://doi.org/10.1037/a0019096

Hess, T. M., Strough, J., \& Löckenhoff, C. (2015). Aging and Decision Making: Empirical and Applied Perspectives. Academic Press.

Josef, A. K., Richter, D., Samanez-Larkin, G. R., Wagner, G. G., Hertwig, R., \& Mata, R. (2016). Stability and Change in Risk-Taking Propensity Across the Adult Life Span. Journal of Personality and Social Psychology, 111(3), 430-450. https://doi.org/10.1037/pspp0000090

Kaiser, T., \& Menkhoff, L. (2017). Does Financial Education Impact Financial Literacy and Financial Behavior, and If So, When? The World Bank Economic Review, 31(3), 611630.

Kircanski, K., Notthoff, N., DeLiema, M., Samanez-Larkin, G. R., Shadel, D., Mottola, G., Carstensen, L. L., \& Gotlib, I. H. (2018). Emotional arousal may increase susceptibility to fraud in older and younger adults. Psychology and Aging, 33(2), 325-337. https://doi.org/10.1037/pag0000228

Ko, K. J., \& Rapkin, S. (2018). The Financial Illiteracy and Overconfidence of Margin Traders. U.S. Securities and Exchange Commission. https://www.sec.gov/dera/staff-papers/whitepapers/dera_wp_financial_illiteracy_and_overconfidence 
Kovalchik, S., Camerer, C. F., Grether, D., Plott, C., \& Allman, J. M. (2005). Aging and decision making: A comparison between neurologically healthy elderly and young individuals. Journal of Economic Behavior and Organization.

Li, Y., Baldassi, M., Johnson, E. J., \& Weber, E. U. (2013). Complementary cognitive capabilities, economic decision making, and aging. Psychology and Aging, 28(3), 595613. https://doi.org/10.1037/a0034172

Li, Y., Gao, J., Enkavi, A. Z., Zaval, L., Weber, E. U., \& Johnson, E. J. (2015). Sound credit scores and financial decisions despite cognitive aging. Proceedings of the National Academy of Sciences, 112(1), 65-69. https://doi.org/10.1073/pnas.1413570112

Löckenhoff, C. E., \& Samanez-Larkin, G. R. (2020). Age Differences in Intertemporal Choice: The Role of Task Type, Outcome Characteristics, and Covariates. The Journals of Gerontology Series B: Psychological Sciences and Social Sciences, 75(1), 85-95. https://doi.org/10.1093/geronb/gbz097

Mamerow, L., Frey, R., \& Mata, R. (2016). Risk taking across the life span: A comparison of self-report and behavioral measures of risk taking. Psychology and Aging, 31(7), 711723. https://doi.org/10.1037/pag0000124

Marquié, J. C., \& Huet, N. (2000). Age differences in feeling-of-knowing and confidence judgments as a function of knowledge domain. Psychology and Aging, 15(3), 451-461. https://doi.org/10.1037/0882-7974.15.3.451

Mata, R., Josef, A. K., Samanez-Larkin, G. R., \& Hertwig, R. (2011). Age differences in risky choice: A meta-analysis. Annals of the New York Academy of Sciences, 1235(1), 18-29. https://doi.org/10.1111/j.1749-6632.2011.06200.x

Mather, M. (2006). A review of decision making processes: Weighing the risks and benefits of aging (L. L. Carstensen \& C. R. Hartel, Eds.; pp. 145-173). The National Academies Press.

Minggui, Y. U., Wengui, L. I., \& Hongbo, P. A. N. (2013). Managerial Overconfidence and Corporate Risk-Taking. Journal of Financial Research, 1. http://en.cnki.com.cn/Article_en/CJFDTotal-JRYJ201301014.htm

Niu, J. (2010). The effect of CEO overconfidence on bank risk taking. Economics Bulletin, 30(4), 3288-3299. 
Nosić, A., \& Weber, M. (2010). How Riskily Do I Invest? The Role of Risk Attitudes, Risk Perceptions, and Overconfidence. Decision Analysis, 7(3), 282-301. https://doi.org/10.1287/deca.1100.0178

Odean, T. (1998). Volume, Volatility, Price, and Profit When All Traders Are Above Average. The Journal of Finance, 53(6), 1887-1934.

Okun, M. A. (1976). Adult age and cautiousness in decision: A review of the literature. Human Development, 19(4), 220-233.

Pak, T.-Y., \& Chatterjee, S. (2016). Aging, overconfidence, and portfolio choice. Journal of Behavioral and Experimental Finance, 12, 112-122. https://doi.org/10.1016/j.jbef.2016.10.003

Peters, E., Hess, T. M., Västfjäll, D., \& Auman, C. (2007). Adult Age Differences in Dual Information Processes: Implications for the Role of Affective and Deliberative Processes in Older Adults\&apos; Decision Making. Perspectives on Psychological Science, 2(1), 123.

Pillemer, K., Connolly, M.-T., Breckman, R., Spreng, N., \& Lachs, M. S. (2015). Elder Mistreatment: Priorities for Consideration by the White House Conference on Aging. The Gerontologist, 55(2), 320-327. https://doi.org/10.1093/geront/gnu180

Pliske, R. M., \& Mutter, S. A. (1996). Age Differences in the Accuracy of Confidence Judgments. Experimental Aging Research, 22(2), 199-216. https://doi.org/10.1080/03610739608254007

Rolison, J. J., Hanoch, Y., Wood, S., \& Liu, P.-J. (2013). Risk-Taking Differences Across the Adult Life Span: A Question of Age and Domain. The Journals of Gerontology Series B: Psychological Sciences and Social Sciences, gbt081. https://doi.org/10.1093/geronb/gbt081

Samanez-Larkin, G. R., \& Knutson, B. (2015). Decision making in the ageing brain: Changes in affective and motivational circuits. Nature Reviews Neuroscience, 16(5), 278-289. https://doi.org/10.1038/nrn3917

Seaman, K. L., Leong, J. K., Wu, C. C., Knutson, B., \& Samanez-Larkin, G. R. (2017). Individual differences in skewed financial risk-taking across the adult life span. Cognitive, Affective, \&amp; Behavioral Neuroscience, 17(6), 1232-1241. https://doi.org/10.3758/s13415-017-0545-5 
Serra, M. J., Dunlosky, J., \& Hertzog, C. (2008). Do Older Adults Show Less Confidence in Their Monitoring of Learning? Experimental Aging Research, 34(4), 379-391. https://doi.org/10.1080/03610730802271898

Shao, R., \& Lee, T. M. (2014). Aging and risk taking: Toward an integration of cognitive, emotional, and neurobiological perspectives. Neuroscience and Neuroeconomics. https://doi.org/10.2147/NAN.S35914

Spreng, R. N., Cassidy, B. N., Darboh, B. S., DuPre, E., Lockrow, A. W., Setton, R., \& Turner, G. R. (2017). Financial Exploitation Is Associated With Structural and Functional Brain Differences in Healthy Older Adults. The Journals of Gerontology: Series A, 72(10), 1365-1368. https://doi.org/10.1093/gerona/glx051

Spreng, R. N., Karlawish, J. H. T., \& Marson, D. C. (2016). Cognitive, social, and neural determinants of diminished decision-making and financial exploitation risk in aging and dementia: A review and new model. Journal of Elder Abuse \& Neglect, 28(4-5), 320344. https://doi.org/10.1080/08946566.2016.1237918

Strough, J., Karns, T. E., \& Schlosnagle, L. (2011). Decision-making heuristics and biases across the life span. Annals of the New York Academy of Sciences, 1235(1), 57-74. https://doi.org/10.1111/j.1749-6632.2011.06208.x

Yu, L., Mottola, G., Bennett, D. A., \& Boyle, P. A. (2020). Confidence in financial and health literacy and cognitive health in older persons. Journal of Alzheimer's Disease. 